\title{
Immune checkpoint inhibitor-induced inflammatory arthritis persists after immunotherapy cessation
}

\author{
Tawnie J Braaten, ${ }^{1}$ Julie R Brahmer, ${ }^{2}$ Patrick M Forde, ${ }^{2}$ Dung Le, ${ }^{2}$ Evan J Lipson, ${ }^{2}$ \\ Jarushka Naidoo, ${ }^{2}$ Megan Schollenberger, ${ }^{2}$ Lei Zheng, ${ }^{2}$ Clifton 0 Bingham $3 \mathrm{rd},{ }_{1}{ }^{1}$ \\ Ami A Shah, ${ }^{1}$ Laura C Cappelli (i) ${ }^{1}$
}

\section{Handling editor Josef $S$ Smolen \\ - Additional material is published online only. To view please visit the journal online (http://dx.doi.org/10.1136/ annrheumdis-2019-216109).}

'Rheumatology, Johns Hopkins School of Medicine, Baltimore, Maryland, USA

2Oncology, Johns Hopkins School of Medicine, Baltimore, Maryland, USA

\section{Correspondence to Dr Laura C Cappelli,}

Rheumatology, Johns Hopkins School of Medicine, Baltimore, MD 21224, USA;

Icappel1@jhmi.edu

AAS and LCC contributed equally.

Received 1 August 2019

Revised 10 September 2019

Accepted 12 September 2019

Published Online First

20 September 2019

\section{SLinked}

- http://dx.doi.org/10.1136/ annrheumdis-2019-216510

Check for updates

(C) Author(s) (or their employer(s)) 2020. No commercial re-use. See rights and permissions. Published by BMJ.

To cite: Braaten TJ, Brahmer JR, Forde PM,

et al. Ann Rheum Dis

2020:79:332-338.

\section{ABSTRACT}

Objective We sought to investigate the long-term outcomes of patients who develop immune checkpoint inhibitor (ICI)-induced inflammatory arthritis (IA), to define factors associated with IA persistence after ICI cessation, the need for immunosuppressants and the impact of these medications on underlying malignancies. Methods We conducted a prospective observational study of patients referred for IA associated with ICls. Patients were recruited from June 2015 to December 2018. Information was obtained at the baseline visit, and follow-up visits occurred at varying intervals for up to 24 months from ICl cessation. Kaplan-Meier curves were developed to characterise IA persistence. Cox proportional hazards models were used to assess the influence of various factors on IA persistence. Logistic regression was used to evaluate the impact of $I A$ treatment on tumour response.

Results Sixty patients were monitored with a median follow-up after $\mathrm{ICI}$ cessation of 9 months. A majority (53.3\%) had active IA at their most recent follow-up. IA was less likely to improve in those with longer duration of $\mathrm{ICl}$ use, in those receiving combination $\mathrm{ICI}$ therapy, and in patients with multiple other immune-related adverse events. Tumour response did not appear to be impacted by immunosuppression. Although not statistically significant, persistent IA was correlated with a better tumour response (complete or partial response). Conclusion ICl-induced IA can become a long-term disease necessitating management by rheumatology for immunomodulatory treatment. Importantly, the use of immunomodulatory treatment has not been shown to impact cancer outcomes in this study.

\section{INTRODUCTION}

The use of immune checkpoint inhibitor (ICI) therapy is rapidly growing, and new agents continue to be approved by the US Food and Drug Administration and European Medicines Agency. ${ }^{12}$ An antiCTLA4 agent, ipilimumab, was first approved for metastatic melanoma in 2011. Since then, approvals have been granted for: antiprogrammed death receptor-1 (PD-1) and anti-PD ligand-1 (PDL-1) medications. ${ }^{3}$ These agents are approved to treat an increasingly wide variety of cancers, both in the metastatic and adjuvant settings. ${ }^{1}$

While ICIs have improved overall survival and cancer progression-free duration, they can be associated with various autoimmune and inflammatory syndromes known as immune-related adverse

\section{Key messages}

- Immune checkpoint inhibitor (ICI)-induced inflammatory arthritis (IA) may persist after ICI cessation.

- Longer $\mathrm{ICl}$ exposure, receipt of combination $\mathrm{ICI}$ therapy and a history of other immunerelated adverse events increase the risk of IA persistence.

- Immunomodulatory treatments were efficacious for symptom control while having no apparent effect on tumour response at follow-up.

- Persistent arthritis may be associated with better tumour response (complete or partial response).

events (irAEs). ${ }^{4}$ These events are thought to occur through the non-specific activation of T-cells against 'self' antigens. ${ }^{5}$ Some irAEs, such as colitis and pneumonitis, can be life-threatening, while others such as inflammatory arthritis (IA) can have a dramatic impact on quality of life. IA is often under-recognised likely due to its limited impact on mortality, examination findings that may miss the threshold of detection by oncology providers, and a broad range of classification options in the Common Terminology Criteria for Adverse Events grading used in clinical trials. However, the necessity for early recognition of IA is growing due to patient functional loss, reports of rapid development of erosions and persistence of joint symptoms. ${ }^{6-10}$ The true incidence of IA resulting from irAEs is difficult to determine, but up to $43 \%$ of patients in immunotherapy clinical trials reported arthralgias, and it has been estimated that between $3.0 \%$ and $7.5 \%$ of patients treated with ICIs develop IA. ${ }^{11-14}$ In light of the percentage of patients who develop IA and the growing use of ICI therapy, further evaluation of long-term outcomes is warranted, particularly in the setting of improved survival on ICI treatment and previous observations that IA persists after ICI cessation. $^{7-10}$

Little is known about risk factors for persistent IA, appropriate treatment strategies in these patients, or the potential impact of immunosuppressive therapy on tumour response. The treatment of IA is challenging in that individuals may require immunosuppression but have recently received an immune-activating agent. Previous studies note that a majority of patients required 
systemic corticosteroids for treatment of IA, and of those 15\%-90\% require additional immunosuppression with conventional synthetic (CS) disease-modifying antirheumatic drugs (DMARDs) or biological (b) DMARDs including tumour necrosis factor (TNF)-inhibitors and interleukin-6 inhibition. ${ }^{8915-17}$ One study reported no impact of non-corticosteroid immunosuppression on existing antitumour responses in patients with ICIrelated IA. ${ }^{8}$

This study investigated the long-term outcomes of patients who developed IA associated with ICI therapy. We focused on those who developed persistent symptoms after ICI cessation and further evaluated whether factors such as combination ICI therapy, ICI exposure duration or the development of other irAEs were predictive of ongoing IA. Lastly, we assessed if immunosuppressive treatment for IA had an impact on the underlying tumour response to ICIs.

\section{MATERIALS AND METHODS \\ Patients}

This is a prospective observational study of patients referred to the Johns Hopkins Arthritis Center for IA due to ICIs. Patients were recruited from June 2015 to December 2018. Patients were included if they were age 18 or older, had received treatment with an ICI (anti-CTLA4, anti-PD1, anti-PDL-1 or combination ICI therapy) and had a rheumatologist-confirmed diagnosis of IA. We excluded patients if they had a pre-existing rheumatological autoimmune disease, were actively receiving ICI therapy or restarted ICI therapy during follow-up, or if they were enrolled in a trial involving investigational agents for which results were not published.

\section{Patient involvement}

Patients with ICI-induced IA were not formally involved in the design for this study. However, discussions with patients by the authors emphasised the importance to patients of understanding whether IA would persist and how likely this would be, the key questions of this study.

\section{Clinical measures}

Baseline data including demographics, cancer type, specific ICI treatment, tumour response, personal and family history of autoimmunity, other irAEs, labs (rheumatoid factor (RF), anticyclic citrullinated peptide (CCP), antinuclear antibody (ANA), erythrocyte sedimentation rate (ESR), $C$ reactive protein (CRP) and clinical examination findings (28-tender joint count, 28 -swollen joint count, dactylitis and/or enthesitis) were obtained at the initial visit. Follow-up occurred at varying intervals for up to 24 months after ICI cessation to assess the clinical status of IA and malignancy. IrAE information was obtained through medical records review. Tumour response was determined by Response Evaluate Criteria in Solid Tumours 1.1 (RECIST) when available. ${ }^{18}$ Otherwise, tumour response was as documented by the patient's treating oncologist in the medical record (ie, in the assessment portion of the progress note or imaging reports). Tumour response was recorded at follow-up visits. Active arthritis was defined as the presence of joint disease (synovitis, tender joints, enthesitis and dactylitis) based on rheumatologist examination or the inability to taper immunosuppressive therapy without return of IA symptoms. If patients had no evidence of active IA at around 6 months after ICI cessation, DMARD medications were tapered. Patients were treated with non-steroidal anti-inflammatory drugs (NSAIDs), intra-articular steroids, systemic steroids, csDMARDS (methotrexate (MTX), leflunomide, sulfasalazine, hydroxychloroquine (HCQ) and bDMARDs (infliximab, adalimumab and etanercept).

\section{Statistics}

Descriptive statistics were calculated for baseline characteristics. The distributions of variables were analysed; median values are presented as they were more representative of the distribution of data for the cohort. Kaplan-Meier curves were used to evaluate the persistence of arthritis over time and the influence of various features on persistent arthritis. The time origin for survival analysis was the date of ICI cessation with follow-up occurring over 24 months from ICI cessation. The event of interest was resolution of IA. Log rank testing was performed to evaluate for significant differences when comparing survival curves. Univariable and multivariable Cox proportional hazards regression was performed to identify factors that associated with IA persistence with the outcome being time to IA resolution. Due to the large number of tumour types observed, only the most common, melanoma and non-small cell lung cancer (NSCLC), were assessed as covariates in the Cox proportional hazards model. Variables with less than three events were not examined as covariates in the multivariable model. Factors that achieved a $\mathrm{p}<0.10$ were included in the multivariable model. Logistic regression analysis was also performed to evaluate the impact of immunosuppression on tumour progression. HRs and ORs were estimated with their 95\% CIs. Statistical significance was set at a $\mathrm{p}<0.05$. All statistical analyses were performed using Stata/IC software V.15.0.

\section{RESULTS}

\section{Baseline patient characteristics}

A total of 60 patients, 32 female and 28 male, were monitored with a median follow-up of 9 months and average follow-up of 12 months (range 1-24 months) after ICI cessation (table 1). The median age was 58.5 years. Of the 60 patients, two had a personal history of single organ autoimmune disease (psoriasis, hypothyroidism) and seven had family history of autoimmune disease including rheumatoid arthritis, Crohn's disease, juvenile idiopathic arthritis, polymyalgia rheumatica and ankylosing spondylitis. The patients had a wide range of cancer diagnoses with melanoma being the most common (35\%), followed by NSCLC (23\%). Gastrointestinal cancers made up 12\% of the study population including pancreatic adenocarcinoma, colon adenocarcinoma, hepatocellular carcinoma and duodenal adenocarcinoma. Four patients had genitourinary cancers (renal cell carcinoma, prostate adenocarcinoma and urothelial carcinoma) and three patients had cervical squamous cell carcinoma, clear cell endometrial carcinoma or endometrial carcinosarcoma. Other cancer diagnoses included Hodgkin's lymphoma, Kaposi sarcoma, mesothelioma, mycosis fungoides, neuroendocrine carcinoma, ependymoma, ductal carcinoma of the breast, basal cell carcinoma and cutaneous squamous cell carcinoma. Combination therapy (anti-CTLA-4+anti-PD-1) was used in 30\% of patients whereas monotherapy with anti-CTLA-4, anti-PD- 1 or anti-PDL-1 was used in 70\% of patients. Some patients received multiple agents over time. ICI treatment was stopped for disease progression, treatment completion and/or severe irAEs. Fourteen $(23 \%)$ had a complete response based on RECIST, imaging reports or treating oncologist documentation.

\section{Baseline features of IA and other irAEs}

The median baseline 28 swollen joint count was 6, and the median baseline 28 tender joint count was 2 (table 2). Median 


\begin{tabular}{|c|c|}
\hline & Values ${ }^{*}, n=60$ \\
\hline \multicolumn{2}{|l|}{ Demographics } \\
\hline Female sex, $(\%)$ & $32(53.3)$ \\
\hline Age (years) & $58.5(52,68)$ \\
\hline BMI $\left(\mathrm{kg} / \mathrm{m}^{2}\right)$ & $26.6(22.6,32.6)$ \\
\hline Race, $(\%)$ & $\begin{array}{l}\text { White: } 54 \text { (90) } \\
\text { Black: } 2 \text { (3.3) } \\
\text { Asian: } 2 \text { (3.3) } \\
\text { White/Asian: } 1 \text { (1.7) } \\
\text { White/Pacific Islander: } 1 \text { (1.7) }\end{array}$ \\
\hline Ethnicity, (\%) & Non-Hispanic: 60 (100) \\
\hline $\begin{array}{l}\text { Personal history of autoimmune disease, } \\
(\%)\end{array}$ & $\begin{array}{l}\text { Psoriasis: } 1 \text { (1.7) } \\
\text { Hypothyroidism: } 1 \text { (1.7) }\end{array}$ \\
\hline $\begin{array}{l}\text { Family history of autoimmune disease, } \\
(\%)\end{array}$ & $\begin{array}{l}\text { Crohn's disease: } 2 \text { (3.3) } \\
\text { Rheumatoid arthritis: } 2 \text { (3.3) } \\
\text { Ankylosing spondylitis and Crohn's } \\
\text { disease: } 1 \text { (1.7) } \\
\text { Juvenile idiopathic arthritis: } 1 \text { (1.7) } \\
\text { Polymyalgia rheumatica: } 1 \text { (1.7) }\end{array}$ \\
\hline Total ICI duration (months) & $7(2,13)$ \\
\hline Follow-up from ICl cessation (months) & $9(5,20.5)$ \\
\hline Tumour type, $(\%) \dagger$ & $\begin{array}{l}\text { Melanoma: } 21(35) \\
\text { NSCLC: } 14 \text { (23.3) } \\
\text { Other: } 11 \text { (18.3) } \\
\text { Gastrointestinal: } 7 \text { (11.7) } \\
\text { Genitourinary: } 4 \text { (6.7) } \\
\text { Gynaecologic: } 3 \text { (5) }\end{array}$ \\
\hline Immunotherapy, (\%) & $\begin{array}{l}\text { Combination: } 18 / 60(30) \\
\text { Monotherapy: } 42 / 60(70)\end{array}$ \\
\hline Baseline tumour response, $(\%)$ & $\begin{array}{l}\text { CR: } 14(23.3) \\
\text { PR: } 10(16.7) \\
\text { NED: } 4(6.7) \\
\text { SD: } 17(28.3) \\
\text { PD: } 15(25.0)\end{array}$ \\
\hline
\end{tabular}

*Values expressed as number (percentage) or median (IQR) as appropriate. †Gastrointestinal cancers included pancreatic adenocarcinoma, colon adenocarcinoma, hepatocellular carcinoma and duodenal adenocarcinoma. Genitourinary cancers included renal cell carcinoma, prostate adenocarcinoma and urothelial carcinoma. Gynaecological cancers included cervical squamous cell carcinoma, clear cell endometrial carcinoma, and endometrial carcinosarcoma. Other cancers included Hodgkin's lymphoma, Kaposi sarcoma, mesothelioma, mycosis fungoides, neuroendocrine carcinoma, ependymoma, ductal carcinoma of the breast, basal cell carcinoma and cutaneous squamous cell carcinoma. $\mathrm{BMI}$, body mass index; $\mathrm{CR}$, complete response; ICl, immune checkpoint inhibitor; NED, no evidence of disease; NSCLC, non-small cell lung cancer; PD, progressive disease; PR, partial response; SD, stable disease.

Clinical Disease Activity Index was 17.5, indicating moderate disease activity. Overall, there were low rates of seropositivity (RF 1.8\%, CCP 5.5\%, ANA 14.3\%) on laboratory analysis. Median baseline ESR and CRP were $29 \mathrm{~mm} /$ hour and $1.3 \mathrm{mg} / \mathrm{dL}$ respectively. Two patients received csDMARD (MTX, HCQ) for IA prior to their initial evaluation in our clinic. Eighteen (30\%) were receiving steroids with a median prednisone dose equivalent of $10 \mathrm{mg}$ daily at the time of presentation to our clinic. NSAIDs alone had been used in $30 \%$ of patients prior to initial rheumatological evaluation. Thirty (50\%) patients had experienced other non-rheumatic irAEs; rash and colitis were the most common, each in $33 \%$ of patients, but a range of other IRAEs were seen (table 2).

\section{Follow-up IA activity}

A majority (53.3\%) of patients had active arthritis at their last follow-up visit which varied from 1 to 24 months after ICI cessation. Three-month follow-up data after ICI cessation was
Table 2 Baseline features of inflammatory arthritis (IA) and other irAEs

\begin{tabular}{|c|c|c|}
\hline & Values ${ }^{*}, \mathrm{n}=60$ & Range/titers \\
\hline \multicolumn{3}{|l|}{ Laboratories } \\
\hline RF Positive, $n=56,(\%)$ & $1(1.8)$ & $0-152$ \\
\hline CCP Positive, $n=55,(\%)$ & $3(5.5)$ & $37,43,2777$ \\
\hline ANA positive, $n=56,(\%)$ & $8(14.3)$ & $\begin{array}{l}\text { No titer-1 } \\
1: 40-2 \\
1: 80-2 \\
1: 160-2 \\
1: 640-1\end{array}$ \\
\hline$E S R, n=53$ & $29(9,53)$ & $1-120$ \\
\hline CRP (mg/dL), $\mathrm{n}=55$ & $1.3(0.2,5.4)$ & $0.1-15.7$ \\
\hline \multicolumn{3}{|l|}{ Examination, (\%) } \\
\hline Dactylitis & $2(3.3)$ & \\
\hline Enthesitis & $3(5.0)$ & \\
\hline $\mathrm{SJC}^{33}$ & $6(3,11)$ & $0-24$ \\
\hline $\operatorname{TJC}^{33}$ & $2(1,4.5)$ & $0-28$ \\
\hline Patient global, $n=44$ & $40(20,72.5)$ & $0-100$ \\
\hline MD global, $n=58$ & $27.5(15,40)$ & $0-80$ \\
\hline CDAl, $n=45$ & $17.5(12,23)$ & $3-56$ \\
\hline $\begin{array}{l}\text { Baseline arthritis } \\
\text { medications, (\%) }\end{array}$ & $\begin{array}{l}\text { Infliximab (for colitis), MTX, and } \\
\text { steroid: } 1 \text { (1.7) } \\
\text { HCQ: } 1 \text { (1.7) } \\
\text { Steroid alone: } 18 / 60(30) \\
\text { Baseline NSAIDs: } 18 / 60(30)\end{array}$ & \\
\hline $\begin{array}{l}\text { Baseline prednisone } \\
\text { equivalent dose (mg) }\end{array}$ & $10(5,50)$ & $2.5-180$ \\
\hline Other irAEs, $(\%)$ & $30(50.0)$ & \\
\hline Specific irAEs, $n=30,(\%)$ & $\begin{array}{l}\text { Rash: } 10(33.3) \\
\text { Colitis: } 10(33.3) \\
\text { Thyroid: } 8 \text { (26.7) } \\
\text { Sicca: } 7 \text { (23.3) } \\
\text { Pneumonitis: } 6(20) \ddagger \\
\text { Hepatitis: } 4 \text { (13.3) } \\
\text { Hypophysitis: } 4 \text { (13.3) } \\
\text { Vitiligo: } 3(10) \\
\text { Pancreatitis: } 1 \text { (3.3) } \\
\text { Sinusitis: } 1 \text { (3.3) } \\
\text { Osteitis: } 1 \text { (3.3) } \\
\text { Arrhythmia with reduced EF: } \\
1 \text { (3.3) }\end{array}$ & \\
\hline
\end{tabular}

\footnotetext{
*Values expressed as number (percentage) or median (IQR) as appropriate. tAn additional two patients developed pneumonitis after their IA visit. ANA, antinuclear antibody; CCP, cyclic citrullinated peptide antibody; CDAl, clinical disease activity index; $C R P, C$ reactive protein; $E$, ejection fraction; ESR, erythrocyte sedimentation rate; $\mathrm{HCQ}$, hydroxychloroquine; irAEs, immune-related adverse events; MTX, methotrexate; NSAID, non-steroidal anti-inflammatory drug; RF, rheumatoid factor; SJC, swollen joint count; TJC, tender joint count.
}

available in 51 patients, with 6-month data available on 41 patients (online supplementary figure 1). At 3 months, $70.6 \%$ had active IA; $48.8 \%$ had active IA at 6 months. Among the 20 patients with persistent arthritis at 6 months, 14 continued to have active disease in further follow-up. Kaplan-Meier curves for persistent arthritis showed that those treated with combination immunotherapy and those with $\geq 2$ irAEs were more likely to persist than those treated with monotherapy or those with $<2$ additional irAEs, respectively (figure $1 \mathrm{~A}, \mathrm{~B}$ ). The curves evaluating persistent arthritis by category of tumour response did not have a statistically significant difference, but showed a trend towards more persistent arthritis in those with complete or partial tumour responses (figure 1C). In univariate analysis (table 3), arthritis was less likely to improve in those with longer duration of ICI exposure (HR 0.93, 95\% CI 0.87 to 0.99 ; 
A

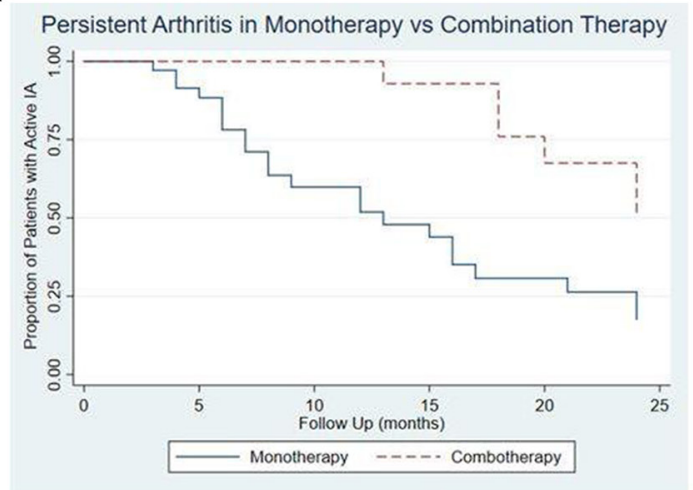

B

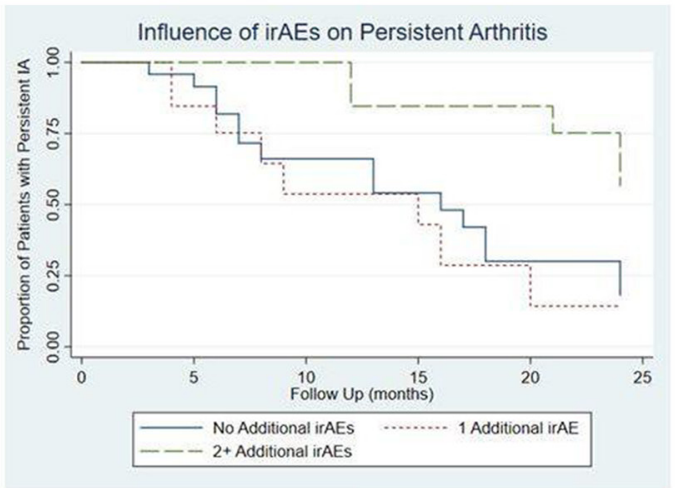

C

Persistent Arthritis and Tumor Response

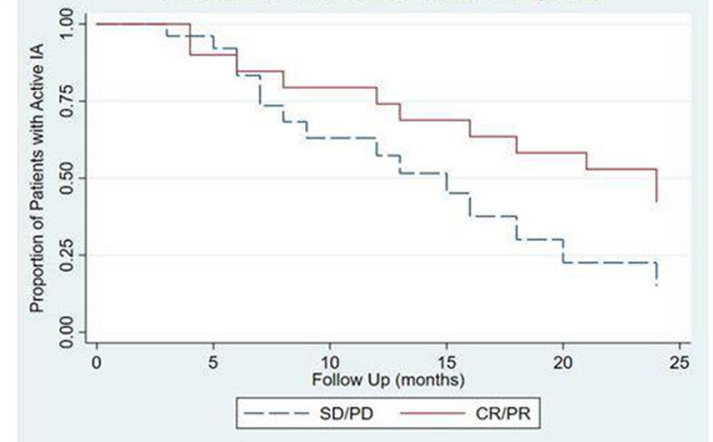

Figure 1 Kaplan-Meier curves for persistent arthritis based on immunotherapy regimen, irAEs, and tumour response. (A) demonstrates increased arthritis persistence in those treated with combination ICI therapy. (B) shows more persistent arthritis in those with $>2$ additional irAEs. (C) shows that patients with a tumour response of complete response or partial response (CR/PR) had more persistent arthritis compared with those with stable disease or progressive disease (SD/PD) at follow-up. IA, inflammatory arthritis; ICI, immune checkpoint inhibitor; irAEs, immune-related adverse events.

$\mathrm{p}=0.02$ ), in those receiving combination ICI therapy (HR 0.29, $95 \%$ CI 0.12 to $0.72 ; \mathrm{p}=0.008$ ) and in patients with history of other irAEs (HR $0.61,95 \% \mathrm{CI} 0.39$ to $0.95, \mathrm{p}=0.03$ ). Duration of ICI treatment (HR $0.82,95 \%$ CI 0.73 to $0.92 ; p=0.001$ ) and combination ICI therapy (HR $0.06,95 \%$ CI 0.01 to 0.50 ; $\mathrm{p}=0.009$ ) remained significant in multivariable analysis (table 4). Although not statistically significant, persistent arthritis was associated with a positive antitumour response rather than stable or progressive disease (HR $0.50,95 \%$ CI 0.22 to $1.11, \mathrm{p}=0.09$; table 3).

\section{IA treatment and impact on tumour response}

Overall, immunomodulatory treatment was required in $75 \%$ of patients to treat the arthritis. Forty-eight patients (80\%) were treated with systemic and/or intraarticular steroids. csDMARDs were used in 19 patients, and bDMARDS were required in 11 (online supplementary table 1 ). Of the 24 patients treated with csDMARDs, bDMARDs or combination of both, 4 (16.7\%) had progression of their cancer during follow-up evaluation, which was not significantly different than tumour progression in 8 (22.2\%) of 36 patients who did not receive DMARDs (OR 0.65 , CI 0.17 to 2.47 ). Of patients who progressed while on DMARDs, one patient was on HCQ, one was on MTX and two were on adalimumab (one for 2 months, the other for 6 months). Adalimumab was stopped in both individuals once cancer progression was noted. In this limited sample size, there was not a statistically significantly increased odd of tumour progression with use of immunosuppressive medications from any category (table 5).

\section{DISCUSSION}

ICI-induced IA is an irAE with the potential to become a chronic disease as demonstrated by the persistence of disease at 3 and 6 months after ICI discontinuation. Factors associated with IA persistence included use of combination ICI therapy, longer duration of ICI exposure and prior development of other irAEs. This information provides insight into which individuals are at highest risk for developing persistent IA, thus warranting close monitoring for joint-related symptoms, early referral to and close follow-up by rheumatology and potentially more aggressive immunosuppressive therapy.

That IA can remain active for months to years after ICI cessation is a significant confirmation of previous reports from smaller series, ${ }^{7-10}$ especially given the paucity of published data on the persistence of other irAEs. Though there are case reports of colitis relapsing after cessation of ICIs ${ }^{19}$ most gastrointestinal irAEs resolve within 3 months of first symptoms and have minimal risk of recurrence. ${ }^{20}$ In contrast, another irAE that may recur or become chronic is pneumonitis; in one cohort, 3 of 19 patients with pneumonitis developed recurrent episodes despite remaining off ICIs. ${ }^{21}$ In a recent poster presentation of six patients with persistent irAEs requiring immunosuppression 6 months after ICI cessation, two had arthritis, and one each had hepatitis, colitis, dermatitis and neuropathy. ${ }^{22}$ Delayed initial presentation of irAEs, with symptoms starting even after ICI cessation, is a related concept that is being increasingly recognised. ${ }^{23}$ Further prospective cohort studies are needed to determine which irAEs besides IA are likely to persist or present after ICI cessation. The types of irAEs that persist may indicate 
Table 3 Cox proportional hazards model: univariable analysis to evaluate factors associated with arthritis persistence

\begin{tabular}{llll}
\hline & HR & 95\% Cl & P value* \\
\hline Female sex & 0.86 & 0.40 to 1.85 & 0.70 \\
\hline Age (years) & 1.02 & 0.99 to 1.05 & 0.12 \\
\hline BMI $\left(\mathrm{kg} / \mathrm{m}^{2}\right.$ ) & 0.95 & 0.88 to 1.02 & 0.17 \\
\hline Family history of autoimmunity & 0.55 & 0.13 to 2.31 & 0.41 \\
\hline TJC $^{33}$ & 0.77 & 0.65 to 0.92 & 0.004 \\
\hline SJC $^{33}$ & 1.0 & 0.92 to 1.09 & 1.0 \\
\hline Enthesitis & 0.65 & 0.24 to 1.76 & 0.40 \\
\hline Physician global assessment & 0.97 & 0.95 to 1.00 & 0.06 \\
\hline Patient global assessment & 0.99 & 0.97 to 1.01 & 0.30 \\
\hline CDAl & 0.95 & 0.90 to 1.00 & 0.07 \\
\hline ESR & 1.01 & 0.99 to 1.02 & 0.29 \\
\hline CRP (mg/dL) & 1.02 & 0.92 to 1.13 & 0.73 \\
\hline Duration ICl therapy (months) & 0.93 & 0.87 to 0.99 & 0.02 \\
\hline Combo versus Mono therapy & 0.29 & 0.12 to 0.72 & 0.008 \\
\hline ANA positivity & 1.77 & 0.52 to 5.98 & 0.36 \\
\hline CCP positivity & 1.76 & 0.23 to 13.37 & 0.58 \\
\hline irAEs (0, 1, 2 or more) & 0.61 & 0.39 to 0.95 & 0.03 \\
\hline Melanoma & 0.49 & 0.22 to 1.12 & 0.09 \\
\hline NSCLC & 0.89 & 0.36 to 2.20 & 0.80 \\
\hline Tumour response & 0.50 & 0.22 to 1.11 & 0.09 \\
\hline (CR/PR vs SD/PD) & & & \\
\hline P-Va & & \\
\hline
\end{tabular}

p-values in bold are statistically significant $(<0.05)$.

* The outcome of interest was resolution of IA. Therefore, HRs $<1$ denote factors that associate with persistence of IA

ANA, antinuclear antibody; BMI, body mass index; $C C P$, cyclic citrullinated peptide antibody; CDAl, Clinical Disease Activity Index; CRP, $C$ reactive protein; CR/PR, complete response and partial response; ESR, erythrocyte sedimentation rate; IA, inflammatory arthritis; ICI, immune checkpoint inhibitor; irAEs, immune-related adverse events; NSCLC, non-small cell lung cancer; SD/PD, stable disease and progressive disease; SJC, swollen joint count; TJC, tender joint count.

how the immune system interacts with particular target tissue microenvironments causing a feed forward loop of autoimmunity that becomes independent of ICIs.

Similar to endocrine-related irAEs, ICI-induced IA may require

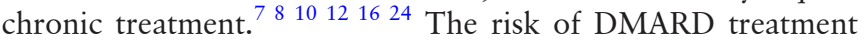
is different than hormone replacement due to the concern that immunosuppression may negatively affect antitumour immune responses. In our small sample size, there was not a change in

Table 4 Cox proportional hazards model: multivariable analysis to evaluate factors associated with arthritis persistence

\begin{tabular}{llll}
\hline & HR & $95 \%$ Cl & P value* \\
\hline TJC $^{3 *}$ & 0.88 & 0.62 to 1.26 & 0.50 \\
MD global assessment & 0.96 & 0.87 to 1.05 & 0.33 \\
CDAl & 1.03 & 0.89 to 1.19 & 0.69 \\
\hline Duration ICI therapy (months) & 0.82 & 0.73 to 0.92 & 0.001 \\
Combo versus monotherapy & 0.06 & 0.01 to 0.50 & 0.009 \\
irAEs (0, 1, 2 or more) & 1.05 & 0.44 to 2.52 & 0.90 \\
Melanoma & 0.41 & 0.10 to 1.78 & 0.24 \\
Tumour response & 1.51 & 0.34 to 6.63 & 0.59 \\
(CR/PR vs SD/PD) & & & \\
\hline
\end{tabular}

Bold values indicate $\mathrm{p}$-values less than 0.05 .

*The outcome of interest was resolution of IA. Therefore, HRs $<1$ denote factors that associate with persistence of IA

CDAl, Clinical Disease Activity Index; CR/PR, complete response and partial response; $I A$, inflammatory arthritis; $I C I$, immune checkpoint inhibitor; irAEs, immune-related adverse events; SD/PD, stable disease and progressive disease; TJC, tender joint count.
Table 5 Univariate analysis: IA treatment and follow-up tumour response (TR)

\begin{tabular}{llllll}
\hline $\begin{array}{l}\text { Medication } \\
\text { exposure }\end{array}$ & $\begin{array}{l}\text { \# of } \\
\text { patients }\end{array}$ & $\begin{array}{l}\text { Worse } \\
\text { TR }^{*}\end{array}$ & OR & $95 \%$ Cl & P value \\
\hline $\begin{array}{l}\text { Systemic steroid } \\
\text { alone }\end{array}$ & 20 & 6 & 2.54 & 0.69 to 9.32 & 0.16 \\
\hline csDMARD & 19 & 2 & 0.34 & 0.07 to 1.74 & 0.20 \\
bDMARD & 11 & 2 & 0.82 & 0.15 to 4.43 & 0.82 \\
Any DMARD & 24 & 4 & 0.65 & 0.17 to 2.47 & 0.53 \\
NSAIDS alone & 7 & 1 & 0.61 & 0.07 to 5.58 & 0.66 \\
\hline
\end{tabular}

*Worse TR: worsened tumour response on follow-up assessment as compared with baseline tumour response (eg, a patient with stable disease at baseline had progression of disease at follow-up).

bDMARD, biological disease-modifying antirheumatic drugs; csDMARD, conventional synthetic disease modifying antirheumatic drugs; IA, inflammatory arthritis; NSAIDs, non-steroidal anti-inflammatory drugs.

tumour response in those treated with csDMARDs or TNF inhibition. This is consistent with previous studies that have shown that treatment of irAEs does not affect tumour response. ${ }^{825}$

This study demonstrated that those treated with combination ICI therapy were more likely to have persistent arthritis. The impact of combination ICI therapy versus monotherapy on irAEs has previously been evaluated. Postow et al reported that severe irAEs were noted in 54\% of patients treated with combination therapy compared with $24 \%$ of those treated with anti-CTLA-4 monotherapy for melanoma. ${ }^{26}$ Larkin et al demonstrated similar findings in melanoma patients with grade 3 or 4 irAEs occurring in $55 \%$ of the nivolumab plus ipilimumab group versus $27.3 \%$ in the ipilimumab alone group and $16.3 \%$ in the nivolumab alone group. ${ }^{27}$ While studies have not evaluated whether IA is more likely to develop in those treated with combination ICI therapy versus monotherapy, previous studies have demonstrated that the type of immunotherapy regimen may influence the type of arthritis, with combination therapy associated with more large joint involvement at onset versus more small joint involvement developing in patients treated with anti-PD-1/PD-L1 monotherapy. ${ }^{8}$ Further evaluation is warranted to examine why the combination of CTLA-4 and PD-1 blockade is more likely to result in large joint involvement and persistent IA disease.

The impact of duration of immunotherapy on specific irAEs is less well defined, as most irAEs occur during the induction period of ICI treatment. ${ }^{24}$ However, various studies have shown that arthritis is a later occurring irAE and can develop months after ICI cessation. ${ }^{7-10}$ 13-16 We demonstrated that individuals treated with ICI therapy for longer duration were more likely to have persistent arthritis at follow-up. The kinetics of ICI-induced IA development differs from many other irAEs and may suggest unique immune pathogenesis for ICI-induced IA, potentially due to longer exposure to ICI therapy.

Appropriate duration of IA treatment remains unknown. One study noted that IA treatment was continued for an average of 9 months after ICI cessation. ${ }^{10}$ In the current report, some patients required immunosuppression $>24$ months after ICI cessation. Since ICI-induced IA is a novel condition, further evaluation is needed to determine the optimal initial treatment, appropriate dose and taper schedule, and duration of therapy.

Interestingly, our data suggest that the development of persistent arthritis may associate with better antitumour responses compared with patients who have transient IA. This could reflect ongoing activation of the immune system that portends better antitumour immunity. Other studies have demonstrated that development of irAEs associates with better 
progression-free and overall survival, but persistence of an irAE has not been examined. ${ }^{128-31}$ In a study of melanoma patients, patients with irAEs of any grade had improved survival compared with those without irAEs. ${ }^{29}$ Studies in non-melanoma patients have also demonstrated similar findings: patients who develop irAEs experience better tumour response. ${ }^{3132}$

The limitations of this study include a possible selection bias for more symptomatic individuals, as it included only patients referred to rheumatology for joint complaints. Less severe cases may have been self-limited or managed by oncology. Also, patients who had persistent IA may have been more likely to pursue longer rheumatology follow-up. A delay in diagnosis may have occurred in the setting of corticosteroids and/or DMARD therapy to manage other irAEs antecedent to the arthritis. Patients were omitted from analysis if they were on a blinded clinical trial or receiving an investigational immunotherapy agent. Additionally, follow-up was limited in some cases due to death in this patient population. Finally, conclusions about the relationship between tumour response and persistent arthritis are limited as not all patients had RECIST scoring. This heterogeneity in monitoring may have under or overestimated the proportion of patients with a positive tumour response.

This study is one of the largest longitudinal reports to date of patients with ICI-induced IA and the first to evaluate persistence of ICI-induced IA and identify influential factors on outcome. Future studies include evaluating genetic risk factors for development of persistent IA. A previous report found that shared epitope alleles were more common in patients with ICI-induced IA; whether this or other genetic factors play a role in IA chronicity is unknown. ${ }^{33}$ Best clinical practices for treating patients with ICI-induced IA based on risk of developing chronic symptoms must also be determined. Those at high risk of developing persistent IA may warrant more specific monitoring approaches by oncologists or preemptive treatments such as HCQ. Another area for inquiry is the differences in timing of development and persistence between IA and other irAEs. Understanding specifics in the biology underlying different irAEs can lead to specifically targeted therapy. Overall, continued clinical and translational investigation on larger longitudinal cohorts will allow for increased understanding of pathophysiology and determination of the best clinical care for patients with ICI-induced IA.

Correction notice This article has been corrected since it published Online First. The author names have been amended.

Acknowledgements We acknowledge Michelle Jones for her contributions as a research coordinator for this manuscript.

Contributors LCC, TJB, AAS and COB III designed the study, acquired and analysed data. All authors were involved in interpretation of data. All authors drafted and edited the manuscript.

Funding The database supporting this study was funded in part through a grant from Bristol-Myers Squibb. Additional support was received from an arthritis fellowship award from AbbVie. Additional support was received from the Camille Julia Morgan Arthritis Research and Education fund, the Jerome L. Greene Foundation, and Core B of P30-AR070354 from the National Institutes of Health (NIH).

Disclaimer These sponsors had no role in study design, data analysis or the decision to publish.

Competing interests Individual sources of potential conflict are as follows: JRB: Consultant/Advisory Boards: Bristol-Myers Squibb, Celgene, Lily Merck, Genentech, Amgen, Janssen, Syndax. Grant/Research Funding: Bristol-Myers Squibb, Merck, Medlmmune/AstraZeneca. PMF: Consultant/Advisory Board: Abbvie AstraZeneca, Boehringer, Bristol-Myers Squibb, Celgene, Eli Lilly, Novartis. Grant/Research Funding: AstraZeneca, BMS, Corvus, Kyowa, Novartis. DL: Consultant/Advisory Board: BristolMyers Squibb, Merck. Grant/Research Funding: Bristol-Myers Squibb, Merck, Aduro Biotech. Honoraria: Merck. EJL: Consultant/Advisory Board: Bristol-Myers Squibb, Novartis, EMD Serono, Array BioPharma, Macrogenics, Merck, Millennium. Grant/
Research Funding: Bristol-Myers Squibb, Merck, Sysmex. Patent/Royalty/Intellectual Property: Method of prevent organ transplant rejection using agonist to the PD-1 checkpoint pathway. JN: Consulting/Advisory Board: AstraZeneca, Roche/Genentech, Bristol-Myers Squibb. Grant/Research Funding: Merck, AstraZeneca. Honoraria: Bristol-Myers Squibb, AstraZeneca. LZ: Consultant/Advisory Boards: Biosynergics, Alphamab, Mingrui, Foundation Medicine, NovaRock Biological, Datareve. Grant/ Research Funding: Halozyme, iTeos, Bristol-Myers Squibb, Merck, Amgen, NovaRock. Licensing Agreement: Aduro Biotech (given up personal royalty). COB III: Consultant/ Advisory Board: Bristol-Myers Squibb, Genentech/Roche, Regeneron/Sanofi. Grant/ Research Funding: Bristol-Myers Squibb. AAS: Consultant/Advisory Board: BristolMyers Squibb. LCC: Consultant/Advisory Board: Regeneron/Sanofi. Grant/Research Funding: Bristol-Myers Squibb.

Patient consent for publication Not required.

Ethics approval This study was approved by the Johns Hopkins Institutional Review Board IRB00123172.

Provenance and peer review Not commissioned; externally peer reviewed. Data availability statement Data are available on reasonable request.

\section{ORCID iD}

Laura C Cappelli http://orcid.org/0000-0003-2795-7059

\section{REFERENCES}

1 Timeline of Progress in Immunotherapy - Cancer Research Institute. Available: https:// www.cancerresearch.org/immunotherapy/timeline-of-progress

2 EMA: European Medical Agency. Available: ema.europa.eu/en

3 The Department of Health and Human Services. Fda approves first treatment for advanced form of the second most common skin cancer, 2018. Available: https:// www.fda.gov/news-events/press-announcements/fda-approves-first-treatmentadvanced-form-second-most-common-skin-cancer-0

4 Weber JS, Kähler KC, Hauschild A. Management of immune-related adverse events and kinetics of response with ipilimumab. JCO 2012;30:2691-7.

5 Pardoll DM. The blockade of immune checkpoints in cancer immunotherapy. Nat Rev Cancer 2012;12:252-64.

6 Albayda J, Dein E, Shah AA, et al. Sonographic findings in inflammatory arthritis secondary to immune checkpoint inhibition: a case series. ACR Open Rheumatology 2019;1:303-7

7 Cappelli LC, Gutierrez AK, Baer AN, et al. Inflammatory arthritis and sicca syndrome induced by nivolumab and ipilimumab. Ann Rheum Dis 2017;76:43-50.

8 Cappelli LC, Brahmer JR, Forde PM, et al. Clinical presentation of immune checkpoint inhibitor-induced inflammatory arthritis differs by immunotherapy regimen. Semin Arthritis Rheu 2018.

9 Calabrese C, Kirchner E, Kontzias K, et al. Rheumatic immune-related adverse events of checkpoint therapy for cancer: case series of a new nosological entity. RMD Open 2017;3:e000412

10 Smith MH, Bass AR. Arthritis after cancer immunotherapy: symptom duration and treatment response. Arthritis Care Res 2019:71:362-6.

11 Cappelli LC, Gutierrez AK, Bingham CO, et al. Rheumatic and musculoskeletal immune-related adverse events due to immune checkpoint inhibitors: a systematic review of the literature. Arthritis Care Res 2017;69:1751-63.

12 Kostine M, Rouxel L, Barnetche T, et al. Rheumatic disorders associated with immune checkpoint inhibitors in patients with cancer-clinical aspects and relationship with tumour response: a single-centre prospective cohort study. Ann Rheum Dis 2018;77:393-8

13 Buder-Bakhaya K, Benesova K, Schulz C, et al. Characterization of arthralgia induced by PD-1 antibody treatment in patients with metastasized cutaneous malignancies. Cancer Immunol Immunother 2018;67:175-82.

14 Lidar M, Giat E, Garelick D, et al. Rheumatic manifestations among cancer patients treated with immune checkpoint inhibitors. Autoimmun Rev 2018;17:284-9.

15 Richter MD, Crowson C, Kottschade LA, et al. Rheumatic syndromes associated with immune checkpoint inhibitors: a single-center cohort of Sixty-One patients. Arthritis Rheumatol 2019:71:468-75.

16 Mooradian MJ, Nasrallah M, Gainor JF, et al. Musculoskeletal rheumatic complications of immune checkpoint inhibitor therapy: a single center experience. Semin Arthritis Rheu 2018.

17 Kim S, Tayar J, Trinh V, et al. Successful treatment of arthritis induced by checkpoint inhibitors with tocilizumab: a case series. Ann Rheum Dis 2016:76:2061-4.

18 Eisenhauer EA, Therasse P, Bogaerts J, et al. New response evaluation criteria in solid tumours: revised RECIST guideline (version 1.1). Eur J Cancer 2009:45:228-47.

19 Kikuchi H, Sakuraba H, Akemoto Y, et al. A case of nivolumab-associated colitis, which relapsed after mucosal healing and was then successfully treated with mesalazine. Immunological Medicine 2019;42:39-44.

20 de Malet A, Antoni G, Collins M, et al. Evolution and recurrence of gastrointestinal immune-related adverse events induced by immune checkpoint inhibitors. Eur J Cancer 2019;106:106-14

21 Asher N, Marom EM, Ben-Betzalel G, et al. Recurrent pneumonitis in patients with melanoma treated with immune checkpoint inhibitors. Oncologist 2019;24:640-7. 
22 Giles CR, Barron C, McWhirter E. Persistent immune-related adverse events after immune checkpoint inhibitor therapy. poster presentation CAMO 2019 annual scientific meeting, 2019. Available: https://www.researchgate.net/publication/ 332929394_Persistent_immune-related_adverse_events_after_immune_checkpoint_ inhibitor_therapy [Accessed 8/19/2019].

23 Couey MA, Bell RB, Patel AA, et al. Delayed immune-related events (dire) after discontinuation of immunotherapy: diagnostic hazard of autoimmunity at a distance. $J$ Immunother Cancer 2019;7.

24 Weber JS, Dummer R, de Pril V, et al. Patterns of onset and resolution of immune-related adverse events of special interest with ipilimumab. Cancer 2013;119:1675-82.

25 Horvat TZ, Adel NG, Dang T-0, et al. Immune-Related adverse events, need for systemic immunosuppression, and effects on survival and time to treatment failure in patients with melanoma treated with ipilimumab at Memorial Sloan Kettering cancer center. JCO 2015;33:3193-8.

26 Postow MA, Chesney J, Pavlick AC, et al. Nivolumab and ipilimumab versus ipilimumab in untreated melanoma. N Engl J Med Overseas Ed 2015;372:2006-17.

27 Larkin J, Chiarion-Sileni V, Gonzalez R, et al. Combined nivolumab and ipilimumab or monotherapy in untreated melanoma. N Engl J Med 2015;373:23-34.
28 Downey SG, Klapper JA, Smith FO, et al. Prognostic factors related to clinical response in patients with metastatic melanoma treated by CTL-Associated antigen-4 blockade. Clinical Cancer Research 2007;13:6681-8.

29 Freeman-Keller M, Kim Y, Cronin $\mathrm{H}$, et al. Nivolumab in resected and unresectable metastatic melanoma: characteristics of immune-related adverse events and association with outcomes. Clinical Cancer Research 2016;22:886-94.

30 Teraoka S, Fujimoto D, Morimoto T, et al. Early Immune-Related Adverse Events and Association with Outcome in Advanced Non-Small Cell Lung Cancer Patients Treated with Nivolumab: A Prospective Cohort Study. J Thorac Oncol 2017; 12:1798-805.

31 Haratani K, Hayashi H, Chiba Y, et al. Association of immune-related adverse events with nivolumab efficacy in Non-Small-Cell lung cancer. Jama Oncol 2017.

32 Judd J, Zibelman M, Handorf E, et al. Immune-Related adverse events as a biomarker in Non-Melanoma patients treated with programmed cell death 1 inhibitors. Oncol 2017;22:1232-7.

33 Cappelli LC, Dorak MT, Bettinotti MP, et al. Association of HLA-DRB1 shared epitope alleles and immune checkpoint inhibitor-induced inflammatory arthritis. Rheumatology 2019;58:476-80. 